\title{
The fast radio burst FRB 20201124A in a star-forming region: Constraints to the progenitor and multiwavelength counterparts
}

\author{
L. Piro ${ }^{1} \odot$, G. Bruni1 ${ }^{1}$, E. Troja ${ }^{2,3}$, B. O’Connor ${ }^{2,3,4,5}$, F. Panessa ${ }^{1}$, R. Ricci ${ }^{6,7}$, B. Zhang ${ }^{8}$, M. Burgay ${ }^{9}$, S. Dichiara ${ }^{2,3}$,
} K. J. Lee ${ }^{10}$, S. Lotti ${ }^{1}$, J. R. Niu ${ }^{11}$, M. Pilia ${ }^{9}$, A. Possenti ${ }^{9,12}$, M. Trudu ${ }^{9}, 12$, H. Xu ${ }^{10}$, W. W. Zhu ${ }^{11}$,

A. S. Kutyrev ${ }^{2,3}$, and S. Veilleux ${ }^{2}$

${ }^{1}$ INAF - Istituto di Astrofisica e Planetologia Spaziali, via Fosso del Cavaliere 100, 00133 Rome, Italy e-mail: luigi.piro@inaf.it

2 Department of Astronomy, University of Maryland, College Park, MD 20742-4111, USA

3 Astrophysics Science Division, NASA Goddard Space Flight Center, 8800 Greenbelt Rd, Greenbelt, MD 20771, USA

${ }^{4}$ Department of Physics, The George Washington University, 725 21st Street NW, Washington, DC 20052, USA

5 Astronomy, Physics, and Statistics Institute of Sciences (APSIS), The George Washington University, Washington, DC 20052, USA

6 INAF - Istituto di Radioastronomia, Via Gobetti 101, 40129 Bologna, Italy

7 Istituto Nazionale di Ricerca Metrologica (INRiM) - Strada delle Cacce 91, 10135 Torino, Italy

8 Department of Physics and Astronomy, University of Nevada, 89154 Las Vegas, NV, USA

9 INAF - Osservatorio Astronomico di Cagliari, via della Scienza 5, 09047 Selargius (CA), Italy

${ }^{10}$ Kavli Institute for Astronomy and Astrophysics, Peking University, Beijing 100871, PR China

11 National Astronomical Observatories, Chinese Academy of Sciences, Beijing 100012, PR China

12 Department of Physics, Università di Cagliari, S.P. Monserrato-Sestu km 0,700, 09042 Monserrato (CA), Italy

Received 29 July 2021 / Accepted 8 November 2021

\section{ABSTRACT}

\begin{abstract}
We present the results of a multiwavelength campaign targeting FRB 20201124A, the third closest repeating fast radio burst (FRB), which was recently localized in a nearby $(z=0.0978)$ galaxy. Deep VLA observations led to the detection of quiescent radio emission, which was also marginally visible in X-rays with Chandra. Imaging at $22 \mathrm{GHz}$ allowed us to resolve the source on a scale of $\gtrsim 1^{\prime \prime}$ and locate it at the position of the FRB, within an error of $0.2^{\prime \prime}$. The EVN and e-MERLIN observations sampled small angular scales, from 2 to 100 mas, providing tight upper limits on the presence of a compact source and evidence for diffuse radio emission. We argue that this emission is associated with enhanced star formation activity in the proximity of the FRB, corresponding to a star formation rate (SFR) of $\approx 10 M_{\odot} \mathrm{yr}^{-1}$. The surface SFR at the location of FRB 20201124A is two orders of magnitude larger than what is typically observed in other precisely localized FRBs. Such a high SFR is indicative of this FRB source being a newborn magnetar produced from a supernova explosion of a massive star progenitor. Upper limits to the X-ray counterparts of 49 radio bursts observed in our simultaneous FAST, SRT, and Chandra campaign are consistent with a magnetar scenario.
\end{abstract}

Key words. stars: magnetars - radio continuum: galaxies - galaxies: star formation $-\mathrm{X}$-rays: bursts

\section{Introduction}

Fast radio bursts (FRBs) are bright radio flashes of millisecond duration (Lorimer et al. 2007; Petroff et al. 2019; Cordes \& Chatterjee 2019) located at cosmological distances. Hundreds of these events have now been discovered (CHIME/FRB Collaboration 2021a), and yet their progenitor systems remain unknown. A leading candidate source is a highly magnetized neutron star (NS), known as a magnetar (Popov \& Postnov 2010; Katz 2016; Metzger et al. 2017; Beloborodov 2017; Kumar et al. 2017; Wadiasingh \& Timokhin 2019; Lyubarsky 2021; Yang \& Zhang 2021), as supported by the recent detection of FRB-like radio bursts from a soft gamma-ray repeater in our galaxy (Scholz \& CHIME/FRB Collaboration 2020; Bochenek et al. 2020; Li et al. 2021; Mereghetti et al. 2020). However, the heterogeneous environment of FRBs and their empirical distinction that spans two classes (repeaters and non-repeaters) leave the door open to multiple progenitor channels (Zhang 2020).

Accurate and rapid localizations of FRBs are key to triggering multiwavelength follow-up observations, which provide vital information on the FRB host galaxy, its distance scale, the broadband spectrum of the burst, and the presence of any quiescent emission associated with the flaring source. However, FRBs span a wide range of distances, and the majority are too far to place any meaningful constraints on persistent emission or associated $\mathrm{X}$-ray bursts. Therefore, we organized a follow-up program to focus on the closest events $(z \lesssim 0.1)$, localized to an accuracy of at least $\lesssim 10^{\prime \prime}$.

Starting from March 21, 2021, the repeating source FRB $20201124 \mathrm{~A}$ was reported to be in an active state (CHIME/FRB Collaboration 2021b; Kumar et al. 2021), with continued activity over the next few months. We initiated our multiwavelength campaign soon after the FRB localization 

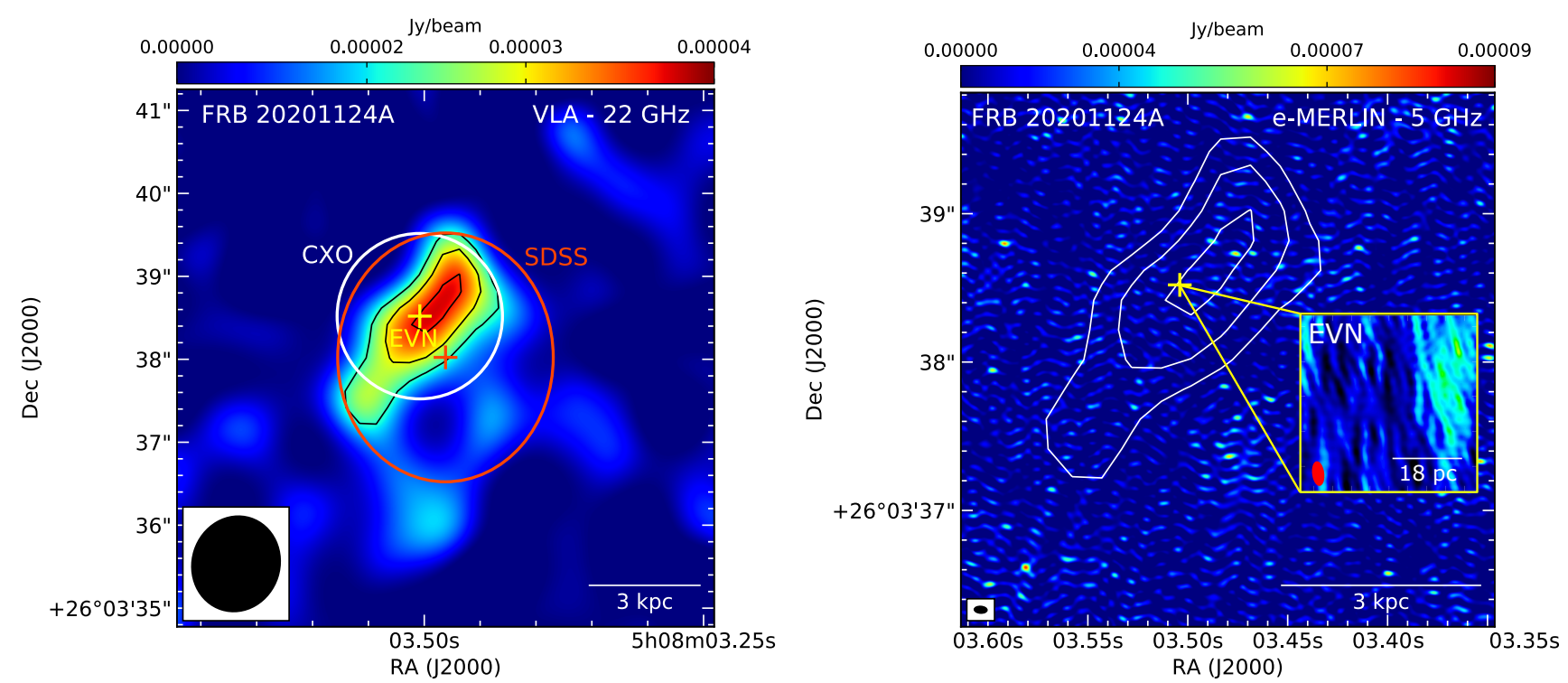

Fig. 1. Imaging and location of FRB 20201124A from radio to X-rays. Left panel: VLA image at $22 \mathrm{GHz}$ presented in this work (in colors), with contours indicating the 3,4, and 5 sigma significance levels. The full width at half maximum is reported in the lower-left corner; shown are the SDSS host galaxy centroid and half-light radius (red) and the CXO extraction region (white), centered on the EVN FRB position (yellow cross). Right panel: e-MERLIN image at $5 \mathrm{GHz}$ of the persistent radio source region, showing no detection. Contours are from the VLA image at $22 \mathrm{GHz}$. The cross indicates the location of FRB 20201124A from EVN observations (Marcote et al. 2021). The full width at half maximum is reported in the lower-left corner. The inset shows the EVN image from our campaign $(25 \times 25$ mas $)$ with no detection at the FRB location, also at $5 \mathrm{GHz}$.

and the unambiguous identification of its host galaxy (Day et al. 2021; Law et al. 2021; Wharton et al. 2021b), which, at a redshift $z \sim 0.098$ (Kilpatrick et al. 2021), meets our selection criteria.

In this paper we present the results of our multi-instrument observations of FRB 20201124A: in radio with the Very Large Array (VLA), the European VLBI Network (EVN), the enhanced Multi Element Remotely Linked Interferometer Network (e-MERLIN), the Five-hundred-meter Aperture Spherical radio Array (FAST), the Sardinia Radio Telescope (SRT), and the upgraded Giant Metrewave Radio Telescope (uGMRT); in X-ray with the Neil Gehrels' Swift Observatory (Swift) and the Chandra X-ray Observatory (CXO); and in the optical with the Lowell Discovery Telescope (LDT; Sect. 2). In Sect. 3 we present the detection of a quiescent source associated with FRB 20201124A in radio and X-rays and discuss its origin. In Sect. 4 we discuss our results in the context of the properties of the local and host galaxy environments and their implications for the progenitors of FRBs, with particular regard to the magnetar scenario. In Sect. 5 we present the results of the simultaneous observation of FAST, SRT, and Chandra, aimed at detecting $\mathrm{X}$-ray counterparts of radio bursts, and discuss implications for the central engine. We summarize our conclusions in Sect. 6.

\section{Observations}

\subsection{Radio}

We searched for a persistent radio source associated with the FRB with the Jansky Very Large Array (JVLA). Observations were carried out under program SG9112 (PI: Piro) at multiple frequencies between April 9 and June 22 (see Appendix A). A radio source, consistent with the FRB position, was detected with a flux of $(340 \pm 30) \mu \mathrm{Jy}$ at $3 \mathrm{GHz}$ (Ricci et al. 2021, see also Wharton et al. 2021a; Ravi et al. 2021). VLA monitoring of this source revealed that the radio emission is not variable, but persistent. However, the initial VLA D configuration did not have sufficient resolution to resolve its angular extent. Therefore, we imaged the field using various beam sizes with the EVN $(\approx 2$ mas $)$, e-MERLIN $(\approx 50-100$ mas $)$, and VLA $\left(\approx 1^{\prime \prime}\right)$. Our EVN and e-MERLIN campaigns (Appendix C) did not detect any compact source over angular scales ranging from 2 mas to 60 mas. At the host redshift of $z \approx 0.098$, this corresponds to a projected linear size of 3 to $110 \mathrm{pc}$ and implies that the persistent radio emission is extended with an angular size $\gtrsim 0.1^{\prime \prime}$, as also independently reported by Marcote et al. (2021) and Ravi et al. (2021).

High-frequency $(22 \mathrm{GHz})$ VLA observations, carried out in $\mathrm{C}$ configuration, partially resolve the source at the scale of $\gtrsim 1^{\prime \prime}$. As shown in Fig. 1, the centroid of the radio emission coincides with the precise FRB position derived by EVN observations (Marcote et al. 2021) within our location accuracy of $0.2^{\prime \prime}$. The radio source is elongated over $\approx 2^{\prime \prime}$ at a position angle of $140^{\circ}$. Its centroid displays marginal evidence of an offset of $0.42^{\prime \prime}$ from the galaxy center, which is more evident when the peak of the radio emission (5 sigma level) is considered. At the redshift of $z=0.0978$ (see Appendix E), the offset of the radio source from the galaxy center would correspond to $0.8 \mathrm{kpc}$ and its extension to $\approx 3 \mathrm{kpc}$.

To extend the frequency coverage at low frequencies, we carried out uGMRT observations on July 21 and 24, 2021 (project code ddtC194; PI: Bruni) using the band-3 (260-500 MHz) and band-2 (125-250 MHz) receivers, respectively. We detected the source as an unresolved component with a flux density of $1.7 \pm 0.2 \mathrm{mJy}$ at $380 \mathrm{MHz}$. The summary of our radio observations is in Table A.1.

\subsection{Optical}

Observations with the Ultra-Violet Optical Telescope (UVOT) on board Swift were performed on April 6, 2021 (PI: Piro, Target ID: 14258) in the $u$ band with a total exposure of $9.9 \mathrm{ks}$. We utilized the uvotimsum task within HEASoft v $\quad 6.27 .2$ to coadd multiple exposures and extracted the photometry with the 
uvotsource tool using a circular aperture of $3^{\prime \prime}$ radius. In the stacked image, we derive an upper limit of $u \gtrsim 22.8 \mathrm{AB}$ mag.

On April 21, 2021, we obtained optical spectroscopy of the putative host galaxy using the DeVeny spectrograph mounted on the $4.3 \mathrm{~m}$ LDT for a total exposure of $4 \times 600 \mathrm{~s}$. DeVeny was configured with the $300 \mathrm{~g} \mathrm{~mm}^{-1}$ grating and a $1.5^{\prime \prime}$ slit width, covering the FRB location. The spectrum covers wavelengths $3600 \AA-8000 \AA$ at a dispersion of $2.2 \AA$ pix $^{-1}$. The resulting spectrum, derived as described in Appendix E, is displayed in Fig. F.2. Emission lines (Table E.1), indicative of ongoing star formation (SF), are detected at $\lambda_{\mathrm{obs}} \approx 7207,7230,7374$, and $7391 \AA$, which we associate with $\mathrm{H} \alpha$, the [NII] doublet, and the [SII] doublet at a redshift of $z=0.0978 \pm 0.0002$, consistent with previous estimates (Ravi et al. 2021; Fong et al. 2021).

\subsection{X-ray}

Observations with the Swift X-ray Telescope (XRT) were carried out as part of the Swift Guest Investigator Program (PI: Piro). Data were collected in photon counting (PC) mode starting on April 6, 2021, for a total exposure of $9.9 \mathrm{ks}$ (Appendix B). No source was detected at the location of the FRB down to a $3 \sigma$ upper limit $<1.3 \times 10^{-3} \mathrm{cts} \mathrm{s}^{-1}(0.3-10 \mathrm{keV})$. We converted this value into an unabsorbed flux $<1.0 \times 10^{-13} \mathrm{erg} \mathrm{cm}^{-2} \mathrm{~s}^{-1}(0.3-$ $10 \mathrm{keV}$ ) assuming an absorbed power law with photon index $\Gamma=2.0$ and a Galactic hydrogen column density $N_{\mathrm{H}}=4.5 \times$ $10^{21} \mathrm{~cm}^{-2}$ (Willingale et al. 2013).

Deeper imaging of the field was carried out with the CXO observations under Director's Discretionary Time (DDT). The observations (ObsID: 25016; PI: Piro) occurred on April 20, 2021, for a total of $29.7 \mathrm{ks}$. Our analysis (Appendix B) was performed in the $0.5-7 \mathrm{keV}$ energy range. A blind search with wavdetect did not find any source at the FRB position; however, a targeted search revealed a weak X-ray detection. We used a circular source region of $1^{\prime \prime}$ radius centered at the EVN position (Marcote et al. 2021) and, within this region, measured a total of three photons. We estimated a background level of $\lesssim 0.25$ cts from nearby source-free regions and a source significance of $99.7 \%$ ( $3 \sigma$ Gaussian equivalent; Kraft et al. 1991).

The observed $0.5-7.0 \mathrm{keV}$ count rate, $1.0_{-0.6}^{+0.7} \times 10^{-4} \mathrm{cts} \mathrm{s}^{-1}$, was converted into flux using the same absorbed powerlaw model described above. For a photon index $\Gamma=$ 2.0 , the unabsorbed flux in the $2-10 \mathrm{keV}$ band is $F_{\mathrm{X}}=$ $1.3_{-0.8}^{+0.9} \times 10^{-15} \mathrm{erg} \mathrm{cm}^{-2} \mathrm{~s}^{-1}$, corresponding to a luminosity of $L_{\mathrm{X}}=(3.1 \pm 1.9) 10^{40} \mathrm{erg} \mathrm{s}^{-1}$.

\subsection{FRB searches}

We organized simultaneous coverage of the Chandra observation with FAST and SRT with the aim of searching for X-ray counterparts of bursts from FRB 20201124A (Appendix H). The radio facilities covered two separate periods for a total of $60 \%$ of the 8.3 hour Chandra observation. A total of 49 radio bursts, with fluences in the range $0.017-13 \mathrm{Jy} \mathrm{ms}$ and with an average duration of about $19 \mathrm{~ms}$, were observed ( 48 by FAST, 1 by SRT). We do not find any significant coincidences with the three X-ray photons detected by Chandra. The closest FRB-X-ray photon time difference is $314 \mathrm{~s}$. (Fig. 2).

\section{Origin of the radio emission}

We argue that the extended radio source is associated with a star-forming region in the host galaxy and exclude an active
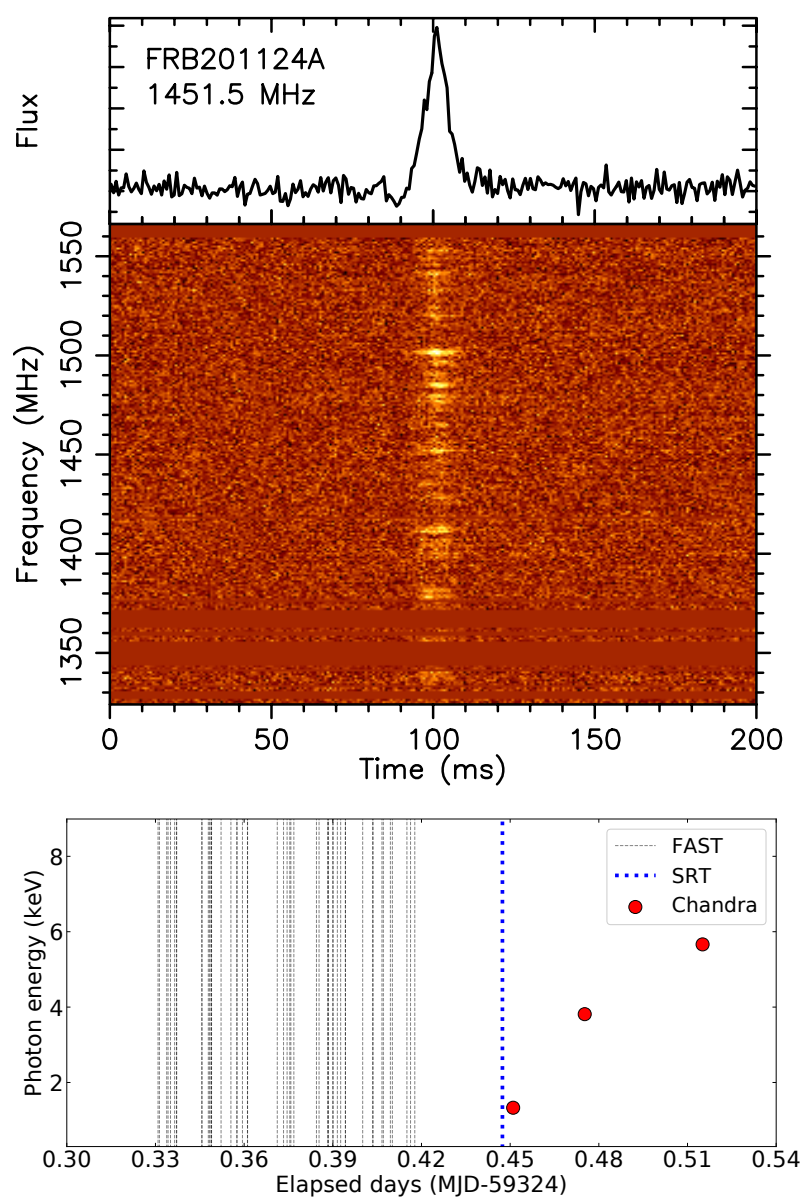

Fig. 2. Search for X-ray counterparts of bursts from FRB 20201124A. Top panel: brightest FRB simultaneous with our Chandra observation, observed by SRT at $T=59324.44738212(12)$ MJD (reported at infinite frequency in Barycentric Dynamical Time units), with a width $=10 \mathrm{~ms}$, a fluence $=13 \mathrm{Jy} \mathrm{ms}$, and $\mathrm{DM}=421 \pm 4 \mathrm{pc} \mathrm{cm}^{-3}$. Bottom panel: arrival times of FRBs detected by FAST (gray lines) and SRT (blue line) compared to the arrival times of Chandra photons (red circles) coincident with the FRB position. The X-ray event closest to an FRB occurs $314 \mathrm{~s}$ after the SRT detected FRB.

galactic nucleus (AGN) origin, as discussed in Appendix G (see also Ravi et al. 2021; Fong et al. 2021). Radio emission from $\mathrm{SF}$ in normal and starburst galaxies is due to the combination of synchrotron emission from cosmic ray electrons produced in supernova $(\mathrm{SN})$ remnants, which dominates below $\approx 20 \mathrm{GHz}$ and is described by a power law with a slope $\alpha_{S}$, and a flat freefree emission and its associated low-frequency absorption, $v_{\mathrm{ff}}$ abs (see Appendix D). In our source, the spectrum from $0.15 \mathrm{GHz}$ to $22 \mathrm{GHz}$ is well described by a power law with $\alpha_{S}=0.76 \pm 0.07$, with no evidence of low-frequency absorption or thermal emission (Fig. 3). Such a spectrum is consistent with that observed in star-forming galaxies (Tabatabaei et al. 2017; Klein et al. 2018). The star formation rate (SFR) associated with this source can be derived using the standard correlation relating SFR to radio luminosity, $\mathrm{SFR}=\frac{L_{1.4 \mathrm{GHz}}}{1.6 \times 10^{28} \mathrm{erg} \mathrm{s}^{-1} \mathrm{~Hz}^{-1}} M_{\odot} \mathrm{yr}^{-1}$ (Murphy et al. 2011). From the observed flux (Table A.1) and the corresponding luminosity, $L(1.4 \mathrm{GHz})=(1.7 \pm 0.2) \times 10^{29} \mathrm{erg} \mathrm{s}^{-1} \mathrm{~Hz}^{-1}$, we derive $\mathrm{SFR} \approx 10 M_{\odot} \mathrm{yr}^{-1}$.

The X-ray data are also in agreement with emission from a star-forming region. X-ray emission is expected from the population of low-mass X-ray binaries (LMXBs) and high-mass X-ray binaries (HMXBs). By adopting the standard correlations that 


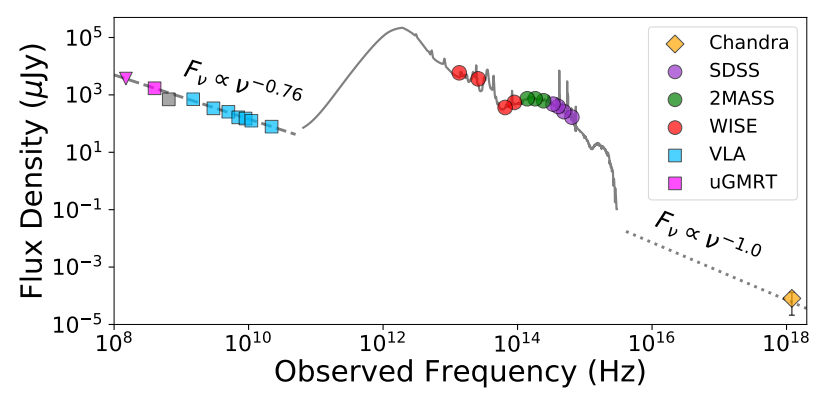

Fig. 3. Broadband radio to X-ray SED of FRB 20201124A. We show the best-fit models to the radio (Appendix D), optical and infrared (Appendix F), and X-ray data (Appendix B) in gray. The dotted line represents the $\mathrm{X}$-ray spectrum expected from the SF derived from the optical SED modeling. It is dominated by a population of HMXBs with a spectrum $\approx v^{-1}$ (Fragos et al. 2013). The downward magenta triangle represents the uGMRT upper limit at $200 \mathrm{MHz}$. The gray square represents the uGMRT data at $650 \mathrm{MHz}$ published by Wharton et al. (2021a).

link SFR to X-ray luminosity (Fragos et al. 2013; Ranalli et al. 2003 ), one expects a $2-10 \mathrm{keV}$ luminosity in the range $L_{X} \approx$ (3-10) $\times 10^{40} \mathrm{erg} \mathrm{s}^{-1}$, consistent with the Chandra measurement (Sect. 2.3 and Appendix B).

Consistent values of the SFR and the key properties of the host galaxy were derived from optical spectroscopy and spectral energy distribution (SED) modeling (Appendices E and F). The best-fit parameters describing the galaxy are: a high intrinsic extinction, $A_{V}=1.3 \pm 0.2 \mathrm{mag}$, symbolic of a dusty galaxy; a sub-solar metallicity, $Z_{\odot}=0.46 \pm 0.25$; an $e$-folding time of $\tau=4.3_{-2.5}^{+3.6} \mathrm{Gyr}$; a moderate stellar mass, $\log \left(M_{*} / M_{\odot}\right)=9.78 \pm 0.08$; an old stellar population, $t_{m}=$ $1.5_{-0.4}^{+0.5} \mathrm{Gyr}$; and an SFR $=4.3 \pm 0.5 \mathrm{M}_{\odot} \mathrm{yr}^{-1}$. The best-fit model spectrum is shown in Fig. 3. From optical spectroscopy, we derive a redshift of $z=0.0978 \pm 0.0002$ and an $\mathrm{H} \alpha$ line luminosity of $L(\mathrm{H} \alpha)=(4.5 \pm 0.8) \times 10^{41} \mathrm{erg} \mathrm{s}^{-1}$, which corresponds to $\mathrm{SFR}_{\mathrm{H} \alpha}=2.3 \pm 0.4 M_{\odot} \mathrm{yr}^{-1}$. As discussed in Appendix E, the $\mathrm{H} \alpha$ luminosity can underestimate the total SFR for observational reasons. Physical reasons can further reduce the $\mathrm{SFR}_{\mathrm{H} \alpha}$ compared to the radio-derived SFR. Radio is also sensitive to heavily obscured SF and, in addition, captures longer star-forming ages, up to $100 \mathrm{Myr}$ (Condon 1992), whereas optical traces recent SF, up to 10 Myr. The mismatch between optical (low SF) and radio (high $\mathrm{SF}$ ) can be interpreted as a contribution from heavily obscured SF or as a post-starburst episode; in other words, $100 \mathrm{Myr}$ ago the SFR was as high as $10 M_{\odot} \mathrm{yr}^{-1}$, but it rapidly declined and within $10 \mathrm{Myr}$ was less than $2 M_{\odot} \mathrm{yr}^{-1}$. A decrease in the SFR at a recent age is also suggested by a non-parametric modelization of the star-forming history (Fong et al. 2021), indicating that the SFR was higher in the past (although at a value lower than the radio-derived SFR) before decreasing by a factor of two $\approx 30 \mathrm{Myr}$ ago. We conclude that the properties of the radio, optical, and X-ray emission are consistent with those expected from SF.

\section{The local star-forming environment as a clue to the progenitor}

The properties of the local environment and of the host of FRBs provide a major clue as to their progenitors. Different progenitor channels are expected to yield distinct distributions of the location, the local environment, and the host galaxy properties (e.g., Margalit et al. 2019). In this regard, FRB 20201124A

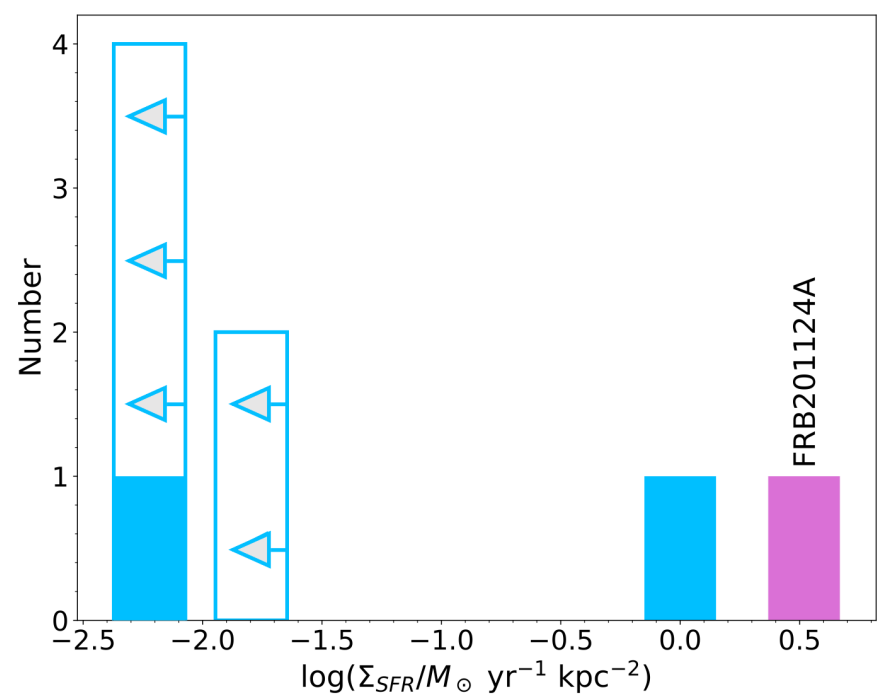

Fig. 4. Local SFR density, $\Sigma_{\text {SFR }}$ (FRB), for FRB 20201124A (this work; magenta bar) compared to the sample of FRBs (from Mannings et al. 2021, with the exception of FRB 20121102A at $1 M_{\odot} \mathrm{yr}^{-1} \mathrm{kpc}^{-2}$, which was derived from Bassa et al. 2017; see text). Arrows represent upper limits, and solid blue bars represent measurements contained in each bin width.

exhibits some remarkable features. We find that it is located, with an accuracy of $360 \mathrm{pc}$, at the center of the most prominent region of SF in the host galaxy, which is observed in the radio with $\mathrm{SFR} \approx 10 M_{\odot} \mathrm{yr}^{-1}$. This structure, elongated by about $3 \mathrm{kpc}$ and at a distance of $0.8 \pm 0.4 \mathrm{kpc}$ from the center of the host galaxy, embeds the FRB and might be associated with a spiral arm. The average SF surface density at the location of the FRB (Fig. 4), $\Sigma_{\mathrm{SFR}}(\mathrm{FRB})=3.3 M_{\odot} \mathrm{yr}^{-1} \mathrm{kpc}^{-2}$, is two orders of magnitude larger than observed in all other FRBs, with the exception of FRB 20121102A, which, with $\mathrm{SFR} \approx 0.2-0.4 M_{\odot} \mathrm{yr}^{-1}$ and a size of $0.68 \mathrm{kpc}$ (Bassa et al. 2017), has $\Sigma_{\mathrm{SFR}}(\mathrm{FRB}) \approx$ $1 M_{\odot} \mathrm{yr}^{-1} \mathrm{kpc}^{-2}$ (see also Mannings et al. 2021), which is comparable to FRB 20201124A. Such large surface SFRs are similar to those observed in Galactic star-forming regions (Evans et al. 2009). This region also exhibits an elevated local SFR in comparison to the mean global value of the host ${ }^{1},\left(\frac{\Sigma_{\mathrm{SFR}}(\mathrm{FRB} 20201124 \mathrm{~A})}{\Sigma_{\mathrm{SFR}}(\text { host })} \gtrsim\right.$ 16), while in most FRBs the local SFR is comparable to the average of the host (Mannings et al. 2021).

The properties of FRB 20201124A, particularly its location within a region of intense SF, favor a prompt formation channel, with the magnetar formed from the SN of a massive star. Systematic studies of host properties (Heintz et al. 2020; Bochenek et al. 2021), including the distribution of stellar masses and SFRs, find a wide range of host properties. These studies concluded that, although this variety can underline a diversity of progenitor channels, the observed global properties are consistent with a predominant population of young magnetars from core-collapse SNe. In this context, the global SFR and stellar mass derived for the host of FRB 20201124A (Fig. F.1) are in agreement with a young magnetar formation from a corecollapse SN.

With regard to the local environment, this progenitor scenario would predict that FRBs should be preferentially located near the brightest star-forming regions of their host 1 Estimated by dividing the total SFR of the galaxy derived from $\mathrm{H} \alpha$
by the area (at half light radius) of the galaxy, $19 \mathrm{kpc}^{2}$. 
galaxies, as we observe in the case of FRB 20201124A. In contrast, Hubble Space Telescope observations of precisely localized FRBs show that while FRB positions are consistent with spiral arms, they are not located in the most active starforming regions (Bhandari et al. 2020; Mannings et al. 2021; Chittidi et al. 2020). On a smaller scale, it is also expected that the positions would be coincident with or at a small offset from star-forming regions. This is due to the limited age $(<10 \mathrm{kyr})$ of the active phase of the magnetar and its kick velocity after birth (e.g., Tendulkar et al. 2021). However, two of the known repeaters, FRB 20180916B and FRB 20121102A, are found at an offset of $\approx 250 \mathrm{pc}$ from the center of the closest star-forming knots, a distance too large to be consistent with a young magnetar progenitor (Bassa et al. 2017; Tendulkar et al. 2021). We find that FRB 20201124A is consistent with being at the center of the SFR, although, given the error in the position, one cannot exclude an offset of $\approx 200 \mathrm{pc}$. In such a case, an age of $0.5 \mathrm{Myr}$ would be expected for a typical kick velocity of $\approx 400 \mathrm{~km} \mathrm{~s}^{-1}$, calling into question a young magnetar origin. In conclusion, the properties of the environment of FRB 20201124A, from the host galaxy scale down to the local environment, are consistent with those expected from a young magnetar progenitor from a SN.

Considering the pieces of evidence discussed above, it is difficult to draw any definitive conclusion on FRB progenitors. Different channels for the formation of magnetar progenitors can explain the broad distribution of properties of the host and local environment. In this scenario FRB 20201124A represents a clear example of a young magnetar progenitor from a corecollapse SN. The recent association of FRB 20200120E with a globular cluster indicates that at least another formation channel is present, with young magnetars produced from the accretioninduced collapse of white dwarfs or binary mergers of NSs (Kirsten et al. 2021). Nonetheless, the fact that the other welllocalized FRBs are close to star-forming regions suggests a predominant channel associated with SF (e.g., Ioka \& Zhang 2020; Tendulkar et al. 2021) but with a broader range of SFRs and offsets, in which FRB 20201124A would represent the extreme in the distribution of the local SFR.

\section{Limits to X-ray counterparts of FRBs}

Notwithstanding the significant progress obtained via the study of the environment, and the increasing number of precise locations that will be available in the future, high resolution imaging of the local environment of FRBs provides only circumstantial evidence regarding the central source of FRBs. A key step forward may come from the identification of the counterpart of FRBs at other wavelengths.

Although our simultaneous coverage of the Chandra observation with FAST and SRT observations does not find any significant association of bursts from FRB 20201124A with X-ray photons (Appendix H), it sets upper limits on the X-ray counterparts that can be confronted with a magnetar origin. We place a $5 \sigma$ upper limit, $E_{\mathrm{X}} \lesssim 6 \times 10^{45} \mathrm{erg}$, in the $2-10 \mathrm{keV}$ range on the X-ray energy of a burst at the time of any radio burst and $E_{\mathrm{X}} \lesssim 1.6 \times 10^{46} \mathrm{erg}$ at any time during the Chandra observation. We derive a tighter upper limit, $E_{\mathrm{X}} \lesssim 1.1 \times 10^{44} \mathrm{erg}$, assuming that X-ray bursts of similar fluence are emitted at the time of each radio burst. In Table H.1 we compare the limits with those of two other repeaters, FRB 20121102A (Scholz et al. 2017) and FRB 20180916B (Scholz et al. 2020). The most constraining limit for X-ray bursts in coincidence with FRB is $E_{\mathrm{X}} \lesssim 1.6 \times 10^{45} \mathrm{erg}$ for a single burst in FRB 20180916B and $E_{\mathrm{X}} \lesssim 1.1 \times 10^{44}$ erg in FRB 20201124A, assuming that all FRBs have an associated X-ray burst. This corresponds to an upper limit on the ratio of the flare energy in X-rays to radio of $E_{\mathrm{X}} / E_{\mathrm{R}} \lesssim 5 \times 10^{5}$, and to a radio-to-X-ray spectral index of $\alpha_{\mathrm{RX}} \gtrsim 0.4\left(F(v) \propto v^{-\alpha}\right)$.

$\mathrm{X}$-ray bursts of known magnetars have isotropic energies much lower than $10^{45} \mathrm{erg}$. The energy of the X-ray burst associated with FRB-like events from the Galactic magnetar SGR $1935+2154$ is $\sim 10^{40} \mathrm{erg}$ (Li et al. 2021). Even for the most energetic giant flares detected from three known magnetars, the isotropic energies only reached a few times $10^{44} \mathrm{erg}$ (Woods \& Thompson 2006). One may imagine more energetic $\mathrm{X}$-ray flares for cosmological FRBs. If we assume that FRBs all have the X-ray-to-radio energy ratio as observed in FRBlike events from the Galactic magnetar SGR 1935+2154 (i.e., $E_{\mathrm{X}} / E_{\mathrm{R}}=3.6 \times 10^{4}$; Bochenek et al. 2020), the upper limit of $E_{\mathrm{X}} / E_{\mathrm{R}} \lesssim 5 \times 10^{5}$ derived from our observations is still consistent with such an assumption. We therefore draw the conclusion that the magnetar flaring origin of FRBs for this source cannot be disfavored and is consistent with the available data.

\section{Conclusions}

In this paper we have presented the results of a campaign, involving nine facilities that operate from radio to X-rays, aimed at constraining the multiwavelength properties of the quiescent and flaring emission of FRB 20201124A. This FRB, which was recently localized, is the third closest repeater.

We find that FRB 20201124A is located at the center of an extended radio source associated with the brightest region of SF in the host galaxy, corresponding to SFR $\approx 10 M_{\odot} \mathrm{yr}^{-1}$. This structure is elongated by about $3 \mathrm{kpc}$ and is at a distance of $0.8 \pm 0.4 \mathrm{kpc}$ from the center of the host galaxy. The SF surface density at the location of the FRB (Fig. 4), $\Sigma_{\mathrm{SFR}}(\mathrm{FRB})=3.3 M_{\odot} \mathrm{yr}^{-1} \mathrm{kpc}^{-2}$, is two orders of magnitude larger than what is typically observed in FRBs (Mannings et al. 2021) but is similar to that observed in Galactic star-forming regions (Evans et al. 2009). This region also exhibits an elevated local SFR in comparison to the mean global value of the host $\left(\frac{\Sigma_{\mathrm{SFR}}(\mathrm{FRB} 20201124 \mathrm{~A})}{\Sigma_{\mathrm{SFR}}(\text { host })} \gtrsim 16\right)$, while in most FRBs the local SFR is comparable to the average of the host. All the properties of the environment of FRB 20201124A, from the host galaxy scale down to the local environment, are consistent with a young magnetar progenitor from a core-collapse SN. In contrast, high resolution observations of the local environment of other precisely located FRBs do not unambiguously support this association (Mannings et al. 2021; Tendulkar et al. 2021), suggesting a diversity of progenitors.

A search for X-ray counterparts of FRBs detected by FAST and SRT, carried out with Chandra, sets upper limits on the $\mathrm{X}$-ray counterparts that can be confronted with a magnetar origin. The tightest upper limit, $E_{\mathrm{X}} \lesssim 1.1 \times 10^{44} \mathrm{erg}$, is derived assuming that X-ray bursts of similar fluence are emitted at the time of each radio flare. This corresponds to an upper limit on the ratio of the flare energy in X-rays to radio of $E_{\mathrm{X}} / E_{\mathrm{R}} \lesssim 5 \times 10^{5}$, consistent with that observed in FRB-like events from the Galactic magnetar SGR 1935+2154 (Bochenek et al. 2020).

Future observations with higher spatial resolution in the optical and radio bands, with adequate sensitivity in radio to low surface brightness, should clarify the morphology of the $\approx 3 \mathrm{kpc}$ star-forming region observed in radio and its association with host galaxy structures, such as spiral arms. Additionally, they should further resolve the region's fine structure such that the 
young magnetar scenario can be tested with a finer measurement of the offset between the FRB and the SFR region.

Acknowledgements. We acknowledge support from a grant PRIN-INAF SKACTA 2016. The National Radio Astronomy Observatory is a facility of the National Science Foundation operated under cooperative agreement by Associated Universities, Inc. We thank the staff of the GMRT that made these observations possible. GMRT is run by the National Centre for Radio Astrophysics of the Tata Institute of Fundamental Research. The European VLBI Network is a joint facility of independent European, African, Asian, and North American radio astronomy institutes. Scientific results from data presented in this publication are derived from the following EVN project code(s): RP032A. e-MERLIN is a National Facility operated by the University of Manchester at Jodrell Bank Observatory on behalf of STFC. We thank the Swift and Chandra staff for the support in carrying out the ToO observations. The Sardinia Radio Telescope (SRT) is funded by the Department of University and Research (MIUR), the Italian Space Agency (ASI), and the Autonomous Region of Sardinia (RAS) and is operated as National Facility by the National Institute for Astrophysics (INAF). We thank an anonymous referee for useful comments.

\section{References}

Aggarwal, K., Agarwal, D., Kania, J. W., et al. 2020, J. Open Source Softw., 5, 2750

Barsdell, B. R., Bailes, M., Barnes, D. G., \& Fluke, C. J. 2012, MNRAS, 422 , 379

Bassa, C. G., Tendulkar, S. P., Adams, E. A. K., et al. 2017, ApJ, 843, L8

Beloborodov, A. M. 2017, ApJ, 843, L26

Bhandari, S., Sadler, E. M., Prochaska, J. X., et al. 2020, ApJ, 895, L37

Biggs, A. D., Younger, J. D., \& Ivison, R. J. 2010, MNRAS, 408, 342

Bochenek, C. D., Ravi, V., Belov, K. V., et al. 2020, Nature, 587, 59

Bochenek, C. D., Ravi, V., \& Dong, D. 2021, ApJ, 907, L31

Bolli, P., Orlati, A., Stringhetti, L., et al. 2015, J. Astron. Instrum., 4, 1550008

Cardelli, J. A., Clayton, G. C., \& Mathis, J. S. 1989, ApJ, 345, 245

Chabrier, G. 2003, PASP, 115, 763

CHIME/FRB Collaboration (Amiri, M., et al.) 2021a, ApJS, submitted [arXiv:2106.04352]

CHIME/FRB Collaboration 2021b, ATel, 14497, 1

Chittidi, J. S., Simha, S., Mannings, A., et al. 2020, ApJ, submitted [arXiv:2005.13158]

Condon, J. J. 1992, ARA\&A, 30, 575

Conroy, C., Gunn, J. E., \& White, M. 2009, ApJ, 699, 486

Cordes, J. M., \& Chatterjee, S. 2019, ARA\&A, 57, 417

Cutri, R. M., Wright, E. L., Conrow, T., et al. 2021, VizieR Online Data Catalog, II/328

Day, C. K., Bhandari, S., Deller, A. T., Shannon, R. M., \& Moss, V. A. 2021, ATel, 14515, 1

Dye, S., Eales, S. A., Aretxaga, I., et al. 2008, MNRAS, 386, 1107

Evans, N. J. I., Dunham, M. M., Jørgensen, J. K., et al. 2009, ApJS, 181, 321

Ferland, G. J., Porter, R. L., van Hoof, P. A. M., et al. 2013, Rev. Mex. Astron. Astrofis., 49, 137

Fong, W.-F., Dong, Y., Leja, J., et al. 2021, ApJ, 919, L23

Fragos, T., Lehmer, B. D., Naoz, S., Zezas, A., \& Basu-Zych, A. 2013, ApJ, 776, L31

Gajjar, V., Siemion, A. P. V., Price, D. C., et al. 2018, ApJ, 863, 2

Grimm, H. J., Gilfanov, M., \& Sunyaev, R. 2003, MNRAS, 339, 793

He, C., Ng, C. Y., \& Kaspi, V. M. 2013, ApJ, 768, 64

Heintz, K. E., Prochaska, J. X., Simha, S., et al. 2020, ApJ, 903, 152

Ibar, E., Ivison, R. J., Biggs, A. D., et al. 2009, MNRAS, 397, 281

Ioka, K., \& Zhang, B. 2020, ApJ, 893, L26

Johnson, B. D., Leja, J. L., Conroy, C., \& Speagle, J. S. 2019, Prospector:

Stellar population inference from spectra and SEDs (Astrophysics Source Code Library)

Kale, R., \& Ishwara-Chandra, C. H. 2021, Exp. Astron., 51, 95

Katz, J. I. 2016, ApJ, 826, 226

Kennicutt, R. C. Jr. 1998, ARA\&A, 36, 189

Kilpatrick, C. D., Fong, W., Prochaska, J. X., et al. 2021, ATel, 14516, 1
Kirsten, F., Marcote, B., Nimmo, K., et al. 2021, ArXiv e-prints [arXiv:2105.11445]

Klein, U., Lisenfeld, U., \& Verley, S. 2018, A\&A, 611, A55

Kraft, R. P., Burrows, D. N., \& Nousek, J. A. 1991, ApJ, 374, 344

Kumar, P., Lu, W., \& Bhattacharya, M. 2017, MNRAS, 468, 2726

Kumar, P., Shannon, R. M., Moss, V., Qiu, H., \& Bhandari, S. 2021, ATel, 14502, 1

Law, C., Tendulkar, S., Clarke, T., Aggarwal, K., \& Bethapudy, S. 2021, ATel, 14526,1

Lehmer, B. D., Basu-Zych, A. R., Mineo, S., et al. 2016, ApJ, 825, 7

Leja, J., Johnson, B. D., Conroy, C., van Dokkum, P. G., \& Byler, N. 2017, ApJ, 837,170

Li, C. K., Lin, L., Xiong, S. L., et al. 2021, Nat. Astron., 5, 378

Licquia, T. C., \& Newman, J. A. 2015, ApJ, 806, 96

Lorimer, D. R., \& Kramer, M. 2004, Handbook of Pulsar Astronomy (Cambridge, UK: Cambridge University Press)

Lorimer, D. R., Bailes, M., McLaughlin, M. A., Narkevic, D. J., \& Crawford, F. 2007, Science, 318, 777

Lyubarsky, Y. 2021, Universe, 7, 56

Mannings, A. G., Fong, W.-F., Simha, S., et al. 2021, ApJ, 917, 75

Marcote, B., Kirsten, F., Hessels, J. W. T., et al. 2021, ATel, 14603, 1

Margalit, B., Berger, E., \& Metzger, B. D. 2019, ApJ, 886, 110

McMullin, J. P., Waters, B., Schiebel, D., Young, W., \& Golap, K. 2007, in Astronomical Data Analysis Software and Systems XVI, eds. R. A. Shaw, F. Hill, \& D. J. Bell, ASP Conf. Ser., 376, 127

Mendel, J. T., Simard, L., Palmer, M., Ellison, S. L., \& Patton, D. R. 2014, ApJS, 210, 3

Mereghetti, S., Savchenko, V., Ferrigno, C., et al. 2020, ApJ, 898, L29

Metzger, B. D., Berger, E., \& Margalit, B. 2017, ApJ, 841, 14

Murphy, E. J., Condon, J. J., Schinnerer, E., et al. 2011, ApJ, 737, 67

O'Connor, B., Troja, E., Dichiara, S., et al. 2021, MNRAS, 502, 1279

Panessa, F., \& Giroletti, M. 2013, MNRAS, 432, 1138

Parma, P., Murgia, M., Morganti, R., et al. 1999, A\&A, 344, 7

Petroff, E., Hessels, J. W. T., \& Lorimer, D. R. 2019, A\&ARv, 27, 4

Popov, S. B., \& Postnov, K. A. 2010, in Evolution of Cosmic Objects through their Physical Activity, eds. H. A. Harutyunian, A. M. Mickaelian, \& Y. Terzian, 129

Prandoni, I., Murgia, M., Tarchi, A., et al. 2017, A\&A, 608, A40

Prescott, M. K. M., Kennicutt, R. C. Jr., Bendo, G. J., et al. 2007, ApJ, 668, 182

Rajwade, K., Stappers, B., \& Williams, C. 2020, in Ground-based and Airborne Instrumentation for Astronomy VIII, eds. C. J. Evans, J. J. Bryant, K. Motohara, et al., International Society for Optics and Photonics (SPIE), 11447,78

Ranalli, P., Comastri, A., \& Setti, G. 2003, A\&A, 399, 39

Ransom, S. M. 2001, PhD Thesis, Harvard University, USA

Ravi, V., Law, C. J., Li, D., et al. 2021, MNRAS, submitted [arXiv:2106. 09710]

Ricci, R., Piro, L., Panessa, F., et al. 2021, ATel, 14549, 1

Saintonge, A., Catinella, B., Cortese, L., et al. 2016, MNRAS, 462, 1749

Schlafly, E. F., \& Finkbeiner, D. P. 2011, ApJ, 737, 103

Schober, J., Schleicher, D. R. G., \& Klessen, R. S. 2017, MNRAS, 468, 946

Scholz, P., \& CHIME/FRB Collaboration 2020, ATel, 13681, 1

Scholz, P., Bogdanov, S., Hessels, J. W. T., et al. 2017, ApJ, 846, 80

Scholz, P., Cook, A., Cruces, M., et al. 2020, ApJ, 901, 165

Shepherd, M.C. 1997, in Astronomical Data Analysis Software and Systems VI, eds. G. Hunt, \& H. Payne, ASP Conf. Ser., 125, 77

Skrutskie, M. F., Cutri, R. M., Stiening, R., et al. 2006, AJ, 131, 1163

Tabatabaei, F. S., Schinnerer, E., Krause, M., et al. 2017, ApJ, 836, 185

Taggart, K., \& Perley, D. A. 2021, MNRAS, 503, 3931

Tendulkar, S. P., Gil de Paz, A., Kirichenko, A. Y., et al. 2021, ApJ, 908, L12

van Dokkum, P. G. 2001, PASP, 113, 1420

Wadiasingh, Z., \& Timokhin, A. 2019, ApJ, 879, 4

Wharton, R., Bethapudi, S., Gautam, T., et al. 2021a, ATel, 14529, 1

Wharton, R., Bethapudi, S., Marthi, V., et al. 2021b, ATel, 14538, 1

Willingale, R., Starling, R. L. C., Beardmore, A. P., Tanvir, N. R., \& O’Brien, P. T. 2013, MNRAS, 431, 394

Woods, P. M., \& Thompson, C. 2006, Soft gamma repeaters and anomalous Xray pulsars: magnetar candidates, 39, 547

Yang, Y.-P., \& Zhang, B. 2021, ApJ, 919, 89

Yang, G., Boquien, M., Buat, V., et al. 2020, MNRAS, 491, 740

Zhang, B. 2020, Nature, 587, 45 


\section{Appendix A: VLA discovery of a quiescent radio source}

Table A.1. Journal of radio observations of the quiescent source associated with FRB 20201124A.

\begin{tabular}{ccccccc}
\hline \hline Telescope & $\begin{array}{c}\text { Date } \\
(\mathrm{dd} / \mathrm{mm} / \mathrm{yyyy})\end{array}$ & $\begin{array}{c}\text { Frequency } \\
(\mathrm{GHz})\end{array}$ & $\begin{array}{c}\text { Bandwidth } \\
(\mathrm{GHz})\end{array}$ & $\begin{array}{c}\text { Time on source } \\
(\mathrm{min})\end{array}$ & $\begin{array}{c}\text { Flux density } \\
(\mu \mathrm{Jy})\end{array}$ & $\begin{array}{c}\text { FWHM } \\
\left({ }^{\prime \prime}\right)\end{array}$ \\
\hline uGMRT & $24 / 07 / 2021$ & 0.19 & 0.125 & 45 & $<3600$ & $21.6 \times 10.4$ \\
uGMRT & $21 / 07 / 2021$ & 0.38 & 0.24 & 98 & $1700 \pm 200$ & $7.0 \times 4.9$ \\
VLA-D & $09 / 04 / 2021$ & 3 & 2 & 28 & $340 \pm 30$ & $18.0 \times 16.1$ \\
& & 9 & 2 & 33 & $150 \pm 10$ & $7.4 \times 6.7$ \\
VLA-D & $11 / 05 / 2021$ & 3 & 2 & 38 & $335 \pm 18$ & $31.7 \times 16.9$ \\
& & 5 & 2 & 11 & $259 \pm 10$ & $20.2 \times 11.4$ \\
& & 7 & 2 & 11 & $165 \pm 11$ & $18.5 \times 8.2$ \\
& & 9 & 2 & 22 & $159 \pm 7$ & $10.4 \times 6.8$ \\
VLA-C & $22 / 06 / 2021$ & 1.5 & 2 & 22 & $126 \pm 10$ & $8.4 \times 5.4$ \\
& & 9 & 2 & 14 & $706 \pm 76$ & $12.8 \times 12.1$ \\
& & 11 & 2 & 10 & $142 \pm 12$ & $2.6 \times 2.6$ \\
& & 22 & 8 & 10 & $104 \pm 14$ & $2.3 \times 2.1$ \\
e-MERLIN & $11 / 07 / 2021$ & 5 & 0.5 & 26 & $78 \pm 8$ & $1.1 \times 1.0$ \\
\hline EVN & $12 / 05 / 2021$ & 5 & $0.1-0.5$ & 245 & $<85$ & $0.085 \times 0.045$ \\
& & & & & $<54$ & $0.0035 \times 0.0017$ \\
\hline
\end{tabular}

We pointed the FRB position from Day et al. (2021) with the VLA on April 9, May 11, and June 22, 2021 (project code SG9112). The first two runs were performed in D configuration and the last in $\mathrm{C}$. The observing bands were $\mathrm{S}(2-4 \mathrm{GHz})$ and $\mathrm{X}$ (8-12 GHz) for the first epoch, S, C (4-8 GHz), and $\mathrm{X}$ for the second one, and $\mathrm{L}(1-2 \mathrm{GHz}), \mathrm{X}$, and $\mathrm{K}(18-26 \mathrm{GHz})$ for the third one (see Table A.1 for a summary). The calibrator 3C 147 was used both for amplitude scale and bandpass corrections, while the phase calibrator paired to the target was J0534+1927 for the first two epochs ( $\sim 9^{\circ}$ apart) and $\mathrm{J} 0431+2037$ for the third one $\left(\sim 10^{\circ}\right.$ apart $)$ due to the configuration change. Data were reduced with the Common Astronomy Software Applications (CASA) package (McMullin et al. 2007). We processed the raw data with pipeline version 5.6.2. The calibrated visibilities were then imaged with the task TCLEAN, with a natural weighting applied to optimize sensitivity.

A constant radio source was detected in the different epochs, offset by $\sim 3.3$ arcsec with respect to the Day et al. (2021) localization but consistent with later ones (Ricci et al. 2021). The flux density was consistent within errors in the different epochs at the common frequencies of 3,9 , and $11 \mathrm{GHz}$ (see Table A.1). The estimated radio luminosity is $L_{5 \mathrm{GHz}}=3 \times 10^{38} \mathrm{erg} \mathrm{s}^{-1}$. The morphology is unresolved at angular resolutions larger than $\sim 1^{\prime \prime}$, while the $22 \mathrm{GHz}$ observations in $\mathrm{C}$ configuration from the latest epoch revealed an elongated emission region, with an extension of $\sim 2^{\prime \prime}$. A Gaussian fit with a single component results in a deconvolved size of $2.2 \pm 0.6 \times 0.5 \pm 0.3^{\prime \prime}$, with a position angle of $140 \pm 8^{\circ}$, confirming the resolved morphology. The component is centered at RA 05:08:03.50 $\pm 0.01 \mathrm{~s}$, Dec $+26: 03: 38.36 \pm 0.18^{\prime \prime}$, consistent with the position of the FRB. The astrometric accuracy was estimated by adopting a conservative VLA positional uncertainty of $10 \%$ of the full width at half maximum ${ }^{2}\left(0.11^{\prime \prime}\right)$ and summing that in quadrature with the peak location error from the Gaussian fit. We obtained a total uncertainty for the Gaussian centroid location of $\pm 0.21^{\prime \prime}$.

\footnotetext{
2 https://science.nrao.edu/facilities/vla/docs/ manuals/oss/performance/positional-accuracy
}

\section{Appendix B: X-ray imaging with Swift and Chandra}

We carried out observations (PI: Piro) with Swift/XRT in PC mode starting at 19:47:02 UT on April 6, 2021, and ending at 14:53:17 UT on April 7, 2021, for a total of $9.9 \mathrm{ks}$ of exposure under ObsIDs 00014258001 and 00014258002 . The data were processed using the xrtpipeline task, and the individual ObsIDs were stacked using XSELECT. The results are presented in Sect. 2.3.

Chandra X-ray Observatory observations (ObsID: 25016; PI: Piro), carried out under DDT, occurred on April 20, 2021, for a total of $29.7 \mathrm{ks}$. Data were processed using the Chandra Interactive Analysis of Observations (CIAO v. 4.13; Fruscione et al. 2006) software and the latest calibration database (CALDB v. 4.9.4). Our analysis was performed in the $0.5-7 \mathrm{keV}$ energy range. The native astrometry of the image was corrected by aligning two common point sources in the Sloan Digital Sky Survey (SDSS) catalog. We restricted these sources to those within 2.5 arcmin of the Chandra/ACIS-S3 detector's optical axis. This resulted in an astrometric shift by $0.5^{\prime \prime}$, with a tie uncertainty of $\sim 0.25^{\prime \prime}$. Further analysis of the astrometric uncertainty using a total of four point sources in common with SDSS, including those at $\gtrsim 2.5$ arcmin from the optical axis of ACIS-S3 sensor, yielded a consistent astrometric solution and did not change the result.

A weak X-ray detection at the position of the EVN verylong-baseline interferometry (VLBI; Marcote et al. 2021) with

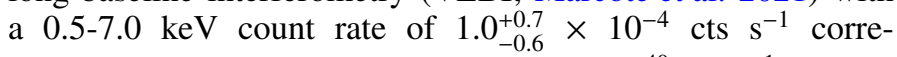
sponds to a luminosity of $L_{\mathrm{X}}=(3.1 \pm 1.9) 10^{40} \mathrm{erg} \mathrm{s}^{-1}$, assuming that absorption is limited to our Galaxy (Sect. 2.3). If we also take into account the large dispersion measure (DM), $421 \pm 4$, associated with the FRB (Appendix $\mathrm{H}$ ), the intrinsic absorption component could be as high as $N_{H, z} \approx 1.2 \times 10^{22}$ $\mathrm{cm}^{-2}$ (He et al. 2013). This would imply an unabsorbed flux of $F_{\mathrm{X}} \approx 1.8 \times 10^{-15} \mathrm{erg} \mathrm{cm}^{-2} \mathrm{~s}^{-1}(2-10 \mathrm{keV})$ and a luminosity of $L_{\mathrm{X}} \approx 4 \times 10^{40} \mathrm{erg} \mathrm{s}^{-1}$.

These values fit well within the correlation between far-infrared and X-ray luminosities in star-forming galaxies 
(Ranalli et al. 2003) and imply a recent SFR $=10_{-7}^{+11} M_{\odot} \mathrm{yr}^{-1}$ (Lehmer et al. 2016). Whereas radio and far-infrared indicators probe the SFR over $\approx 100$ Myr timescales, studies of local star-forming galaxies show that the dominant component of the X-ray emission are HMXBs associated with a young $(\lesssim 30 \mathrm{Myr})$ stellar population (Grimm et al. 2003).

\section{Appendix C: Follow-up of the persistent radio source with EVN, e-MERLIN, and UGMRT}

Following the detection of a persistent radio source with the VLA, we triggered a VLBI campaign involving the EVN and the e-MERLIN. The EVN observations were performed on May 12, 2021, in e-VLBI mode with the standard setup at $5 \mathrm{GHz}$ (project code RP032A, PI: Panessa). The array was composed of nine EVN antennas (IR, BD, SV, HH, SH, O8, EF, WB, and JB) plus six from e-MERLIN (JL, PI, KN, DE, DA, and CM). The data rate was 2 Gbps for all antennas except for SV and BD (1 Gbps) and the e-Merlin array (512 Mbps). This resulted into different bandwidths, from 0.1 to $0.5 \mathrm{GHz}$. Sources J0237+2848 and J0555+3948 were used as fringe finders at the beginning and end of the experiment, while $\mathrm{J} 0502+2516$ was used for target phase referencing $\left(1.4^{\circ}\right.$ apart). A total on-source time of 4 hours was reached. Data were reduced with the Astronomical Image Processing System (AIPS) software, following standard calibration procedures for continuum data. Visibilities were then imaged using the DIFMAP software (Shepherd 1997), with natural weighting applied. The obtained angular resolution was $3.5 \times 1.7$ mas, with a position angle of $7.15^{\circ}$. The RMS was 9 $\mu \mathrm{Jy} /$ beam.

We did not detect any compact source above a confidence level of 6 sigma, resulting in an upper limit of $54 \mathrm{uJy} / \mathrm{beam}$. This result confirms, with deeper observations, the previous one from the PRECISE project (Marcote et al. 2021), also obtained with the EVN but at $1.4 \mathrm{GHz}$. Marcote et al. (2021) concluded that the nature of the emission detected with the VLA, not being visible at VLBI resolution, must be of an extended nature. From non-detections on the shortest baseline of the array (EffelsbergWesterbork; $\sim 270 \mathrm{~km}$ ), they could estimate an angular size of $\gtrsim 140$ mas for the extended emission, corresponding to a projected linear size of $\gtrsim 260 \mathrm{pc}$. In our observation, the sub-array composed of e-MERLIN antennas plus JB (i.e., the array baselines more sensitive to extended emission) gave an angular resolution of $60 \times 36$ mas and an RMS of $43 \mu \mathrm{Jy} / \mathrm{beam}$ (applying natural weighting). Considering a threshold of 6 sigma, the eMERLIN non-detection implies a surface brightness of $<0.045$ $\mathrm{Jy} / \mathrm{kpc}^{2}$.

Given the results of the EVN run, we performed deeper observations with e-MERLIN at $5 \mathrm{GHz}$ on July 11, 2021 (DDT project DD11007, PI: Bruni), with the aim of recovering the SFR emission at an intermediate scale between our previous VLA and EVN observations. The run originally also included $1.5 \mathrm{GHz}$, the frequency band where most of the flux density was expected; however, the sensitivity was hampered by the lack of suitable, unresolved phase calibrators near to target. At $5 \mathrm{GHz}$, we observed with the full $512 \mathrm{MHz}$ bandwidth, for a total of $\sim 11$ hours on target, reaching an RMS of $17 \mu \mathrm{Jy} /$ beam. The phase calibrator was J0506+2141, with an angular separation of $4.4^{\circ}$. The angular resolution, applying natural weighting, was $85 \times 45$ mas. No emission was detected at a significance level higher than 5 sigma at this sensitivity either (see Fig. 1), implying a surface brightness of $<0.008 \mathrm{Jy} / \mathrm{kpc}^{2}$.

To further complete the frequency coverage of the persistent radio source, we requested DDT observations with the uGMRT.
Observations were carried out on July 21 and 24, 2021 (project code ddtC194, PI: Bruni), using the band-3 (260-500 MHz) and band-2 (125-250 MHz) receivers, respectively. The amplitude scale and bandpass calibrations were performed on 3C 147, while the adopted phase calibrator was J0534+1927. Data were reduced with the CAPTURE pipeline (Kale \& Ishwara-Chandra 2021). In band 3 , the final image RMS was $50 \mu \mathrm{Jy} /$ beam and the angular resolution $7.0^{\prime \prime} \times 4.9^{\prime \prime}$. We detected the source as an unresolved component with a flux density of $1.7 \pm 0.2 \mathrm{mJy}$. Band 2 was hampered by radio frequency interferences (RFIs), with more than $50 \%$ of the data flagged on each antenna. The final image showed no detection at an RMS level of $1.2 \mathrm{mJy} / \mathrm{beam}$, resulting in a $3 \sigma$ upper limit of $3.6 \mathrm{mJy}$. The angular resolution was $21.6^{\prime \prime} \times 10.4^{\prime \prime}$.

\section{Appendix D: The radio spectrum from star formation in the host galaxy}

Star-forming galaxies emit both thermal (free-free) and nonthermal (synchrotron) radiation in the radio regime. The synchrotron component, resulting from the $\mathrm{S} / \mathrm{N}$, dominates at frequencies up to about $20 \mathrm{GHz}$, while free-free emission becomes significant at higher frequencies (Klein et al. 2018). At low frequencies, typically well below $1 \mathrm{GHz}$, free-free absorption takes place (Schober et al. 2017). The resulting spectrum is thus described as

$$
F_{v}=C_{S}\left[\frac{v}{G H z}\right]^{-\alpha_{s}} e^{-\tau_{f f}}+C_{f f}\left[\frac{v}{G H z}\right]^{2}\left(1-e^{-\tau_{f f}}\right)
$$

with

$\tau_{f f}(v)=\left[\frac{v}{v_{f f, a b s}}\right]^{-2.1}$

The first term in the equation is the synchrotron component, modeled with a power law with a slope of $\alpha_{s}$. The second, flatter component, $\propto v^{-0.1}$ in the optically thin regime, is due to freefree emission. In Fig. 3 we present the best-fit model of our VLA and uGMRT data (Table A.1). The radio spectrum is dominated by the synchrotron component with $\alpha_{s}=0.76 \pm 0.07$, no evidence of absorption, and an upper limit $(3 \sigma)$ of $\lesssim 10 \%$ of the thermal fraction at $1.4 \mathrm{GHz}$. Those values are consistent with the range observed in the radio spectra of star-forming galaxies (Tabatabaei et al. 2017).

\section{Appendix E: Optical spectroscopy}

We obtained optical spectroscopy of the putative host galaxy of FRB 20201124A on April 21, 2021, starting at 03:18:35.51, with the DeVeny spectrograph mounted on the $4.3 \mathrm{~m}$ LDT for a total exposure of $4 \times 600 \mathrm{~s}$. DeVeny was configured with the $300 \mathrm{~g}$ $\mathrm{mm}^{-1}$ grating and a $1.5^{\prime \prime}$ slit width. The slit was aligned at a position angle of $63^{\circ}$ east of north and covers the location of the FRB as determined by the EVN localization. However, we note that the width of the slit does not completely cover either the full extension of the persistent radio source or the host galaxy. We reduced the data using standard procedures in the Image Reduction and Analysis Facility (IRAF) package to perform bias subtraction, flat field correction, and cosmic ray removal with L. A. Cosmic (van Dokkum 2001).

The spectrum was calibrated using the spectrophotometric standard star Feige 34. The resulting spectrum is displayed in Fig. F.2. We detect emission features at $\lambda_{\mathrm{obs}} \approx 7207,7230$, 7374 , and $7391 \AA$ associated with $\mathrm{H} \alpha$, the [NII] doublet, and 
Table E.1. Host galaxy emission line properties from our optical spectroscopy with LDT/DeVeny. These values are corrected for Galactic extinction, $E(B-V)=0.63 \mathrm{mag}$ (Schlafly \& Finkbeiner 2011).

\begin{tabular}{lcc}
\hline \hline Line & $\lambda_{\text {obs }}$ & Flux \\
\hline & {$[\AA]$} & {$\left[10^{-15} \mathrm{erg} \mathrm{cm}^{-2} \mathrm{~s}^{-1}\right]$} \\
\hline $\mathrm{H} \beta$ & 5345.1 & $<2.9$ \\
$\mathrm{H} \alpha$ & 7206.8 & $7.4 \pm 1.3$ \\
$\mathrm{NII}_{\lambda 6585}$ & 7229.6 & $3.9 \pm 1.1$ \\
SII $_{\lambda 6718}$ & 7373.5 & $2.9 \pm 0.7$ \\
SII $_{\lambda 6732}$ & 7391.3 & $2.2 \pm 0.7$ \\
\hline
\end{tabular}

the $[\mathrm{SII}]$ doublet. Given the sensitivity of these observations, our non-detection of $\mathrm{H} \beta$ or oxygen lines is consistent with the fluxes reported by Fong et al. (2021). Using the observed lines, we derive a redshift of $z=0.0978 \pm 0.0002$. Line properties were derived by fitting the lines with Gaussian functions using the specutils package in Python. The emission line fluxes were corrected for Galactic extinction, $E(B-V)=0.63 \mathrm{mag}$ (Schlafly \& Finkbeiner 2011), assuming a Cardelli et al. (1989) extinction law. The line properties are reported in Table E.1.

From the $\mathrm{H} \alpha$ emission line, we derive an $\mathrm{SFR}_{\mathrm{H} \alpha}=0.89 \pm$ $0.16 M_{\odot} \mathrm{yr}^{-1}$ (Kennicutt 1998), assuming a Chabrier initial mass function (IMF; Chabrier 2003). From the analysis of the host galaxy's SED (Appendix F), we infer a global value of $A_{V} \sim 1.3$ mag for the intrinsic extinction. By applying this correction to our spectrum, the optically derived SFR is $\mathrm{SFR}_{\mathrm{H} \alpha}=2.3 \pm 0.4$ $M_{\odot} \mathrm{yr}^{-1}$.

\section{Appendix F: Galaxy SED analysis}

We modeled the SED of the host galaxy using PROSPECTOR (Johnson et al. 2019) and the methodology outlined in O'Connor et al. (2021). We made use of archival photometry from SDSS, the Two Micron All Sky Survey (2MASS; Skrutskie et al. 2006), and the Wide-field Infrared Survey Explorer (WISE; Cutri et al. 2021). This photometry covers the optical and near-infrared wavelengths ugrizJHK in addition to two WISE infrared bands ( $W 1$ and $W 2$ ). We excluded the WISE $W 3$ and $W 4$ photometry due to the uncertainty surrounding thermal dust emission models (see, e.g., Leja et al. 2017). From the archival SDSS/ $u$-band image, we derive a $3 \sigma$ upper limit $u \gtrsim 22.0 \mathrm{mag}$ on an underlying source. This is consistent with the deeper limit from Swift/UVOT $(u \gtrsim 22.8 \mathrm{mag})$, which we included in our modeling. We also included the measured $\mathrm{H} \alpha$ emission line flux as pseudo narrowband photometry in order to better constrain the SFR. The photometry was corrected for Galactic extinction in the direction of the FRB, $E(B-V)=0.63 \mathrm{mag}$ (Schlafly \& Finkbeiner 2011), prior to modeling with PROSPECTOR. We adopted a Chabrier (2003) IMF with integration limits of 0.08 and $120 M_{\odot}$ (imf_type $=1$ ), an intrinsic dust attenuation using the extinction law of Cardelli et al. (1989) and accounting for both additional dust in nebular regions (dust 1 ) and diffuse dust throughout the galaxy (dust 1$)$, and a delayed $-\tau$ ( $\mathrm{sfh}=4) \mathrm{SF}$ history characterized by an e-folding timescale, $\tau$. We accounted for the contribution of nebular emission using the photoionization code CLOUdY (Ferland et al. 2013). The synthetic SEDs corresponding to these models were computed with the flexible stellar population synthesis (FSPS) code (Conroy et al. 2009). The free parameters in these models are the total stellar mass formed $(M)$, the age of

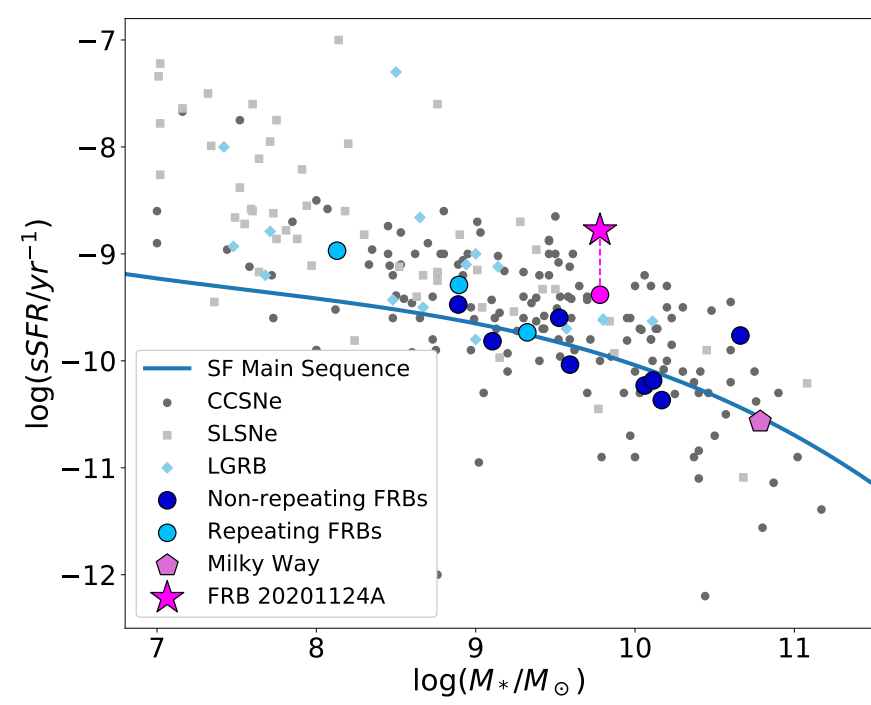

Fig. F.1. Specific SFR (SFR normalized to the total mass of the galaxy) of FRB 20201124A (magenta star), other repeating FRBs (light blue circles), non-repeating FRBs from Heintz et al. (2020) (dark blue circles), and our own Galaxy (light purple pentagon; Licquia \& Newman 2015). For FRB 20201124A we display the specific SFR derived from radio observations (Appendix $\mathrm{A}$ ) with a magenta star, and a dashed line connects this value to the magenta circle representing the specific SFR derived from optical spectroscopy (Appendix E and Fig. F.2). We also display the populations of other astrophysical transients for reference by including the low redshift $(z<0.3)$ populations of long gammaray bursts (LGRBs; small blue diamonds), core-collapse supernovae (CCSNe; small gray circles), and superluminous supernovae (SLSNe; small gray squares) from Taggart \& Perley (2021). These values are compared to the low redshift $(z<0.05)$ star-forming main sequence from Saintonge et al. (2016), displayed by a solid line. Our object is located well above the SF main sequence, above which galaxies have enhanced SF efficiencies.

the galaxy $\left(t_{\text {age }}\right)$, the e-folding timescale $(\tau)$, the intrinsic reddening $\left(A_{V}\right)$, and the metallicity $\left(Z_{*}\right)$. We adopted uniform priors in $\log t_{\text {age }}, \log \tau$, and $\log Z, E(B-V)$ as in Mendel et al. (2014, cf. their Table 2). From these parameters, we derived the stellar mass $\left(M_{*}\right)$, the mass-weighted stellar age $\left(t_{m}\right)$, and the SFR.

The best-fit model spectrum is shown in Fig. 3 and the corner plot in Fig. F.3; the best-fit parameters are reported in Sect. 3. We note that the mass-weighted stellar age and stellar mass are slightly smaller compared to those presented by Ravi et al. (2021) and Fong et al. (2021). We find that this is likely due to different model assumptions within prospector. We compared the stellar mass and SFR to the host galaxies of core-collapse $\mathrm{SNe}$, long gamma-ray bursts, and other FRBs in Fig. F.1.

The X-ray luminosity expected from such a galaxy is derived as follows. There are three main components of galaxy X-ray emission: LMXBs, HMXBs, and hot gas. The strengths of these components can be modeled as a function of galaxy properties, such as $M_{*}$ and SFR. We adopted the recipe from Fragos et al. (2013) and Yang et al. (2020):

$\log \left(L_{2-10 k e V}^{L X R B} / M_{*}\right)=f\left(t_{m}\right)$,

$\log \left(L_{2-10 \mathrm{keV}}^{\mathrm{HXRB}} / S F R\right)=g\left(Z_{*}\right)$,

$\log \left(L_{0.5-2 k e V}^{\text {hot }} / S F R\right)=38.9$, 


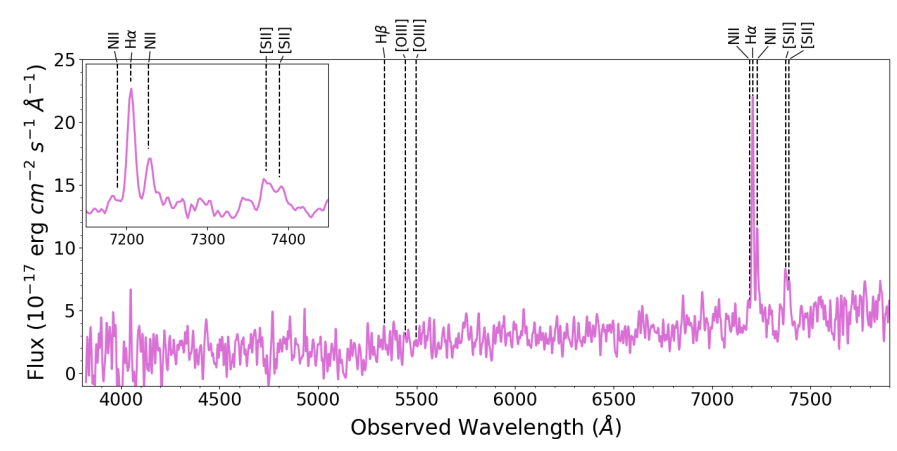

Fig. F.2. LDT/DeVeny optical spectrum of the host galaxy of FRB 20201124A at $z=0.0978 \pm 0.0002$. The spectrum (solid purple line) has been smoothed with a Gaussian kernel. Dashed black lines indicate the expected location of emission lines at this redshift; we do not observe oxygen lines or $\mathrm{H} \beta$. The inset figure shows a zoomed-in view of the region of the detected emission lines between 7150 and $7450 \AA$. The spectrum is not corrected for Galactic extinction.

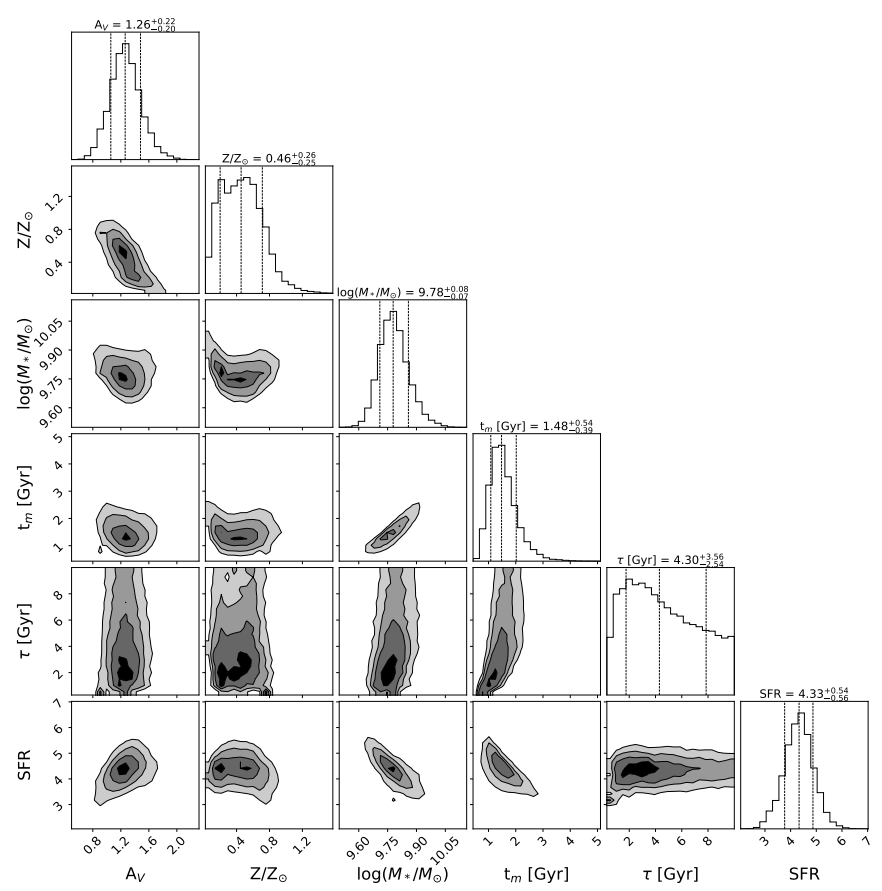

Fig. F.3. Corner plot demonstrating the results of our prospector modeling of the host galaxy SED as outlined in Appendix F.

where luminosities are in erg $\mathrm{s}^{-1}$. The functions $f\left(t_{m}\right)$ and $g\left(Z_{*}\right)$ are given in Fragos et al. (2013). Substituting the best-fit values, one obtains $L_{(2-10 \mathrm{keV})}=(5 \pm 2) \times 10^{40} \mathrm{erg} \mathrm{s}^{-1}$, which is consistent with the observed X-ray luminosity. This emission is dominated $(80 \%)$ by the HMXB component associated with young stellar populations.

\section{Appendix G: Origin of the persistent emission from radio to $\mathrm{X}$-rays}

Our EVN observation demonstrated that the emission is extended and that the bulk of the radio emission must arise on scales larger than about 200 pc. We argue that this emission is produced by SF in the host galaxy. First, the radio luminosity in galaxies is correlated with SFR (Murphy et al.
2011) as $\mathrm{SFR}=\frac{L_{1.4 G H z}}{1.7 \times 10^{28} \mathrm{erg} \mathrm{s}^{-1} \mathrm{~Hz}^{-1}} \mathrm{M}_{\odot} \mathrm{yr}^{-1}$, which, in the case of FRB 20201124A, gives SFR $\approx 10 M_{\odot} y r^{-1}$. This is consistent with the value derived from $\mathrm{X}$-rays and also in agreement with the smaller values derived from the optical and infrared bands (2-4 $\left.M_{\odot} y r^{-1}\right)$, considering that the radio may include additional emission from heavily obscured SF (Prescott et al. 2007; Dye et al. 2008) or from enhanced SF that may have occurred over the longer timescale ( $\lesssim 100 \mathrm{Myr}$ ) probed by radio observations (Condon 1992). Furthermore, the radio spectrum in the 0.4-10 $\mathrm{GHz}$ range has a slope of $0.76 \pm 0.07$, which is consistent with the average of $0.7 \pm 0.4$ observed in star-forming galaxies (Ibar et al. 2009).

Alternative scenarios involving a low-surface-brightness AGN-driven radio emission (e.g., jets) are unlikely (Biggs et al. 2010). A low-luminosity radio-quiet AGN, such as lowionization nuclear emission-line regions and Seyfert galaxies, would be characterized by a more compact morphology (e.g., Panessa \& Giroletti 2013). A small fraction of nuclei active in radio are in the so-called dying or remnant phase. The active phase of a radio AGN can last several tens of megayears, after which the nuclear activity stops and the source starts to fade away (e.g., Parma et al. 1999). The core and the jets disappear and only the lobes remain visible, radiating their energy away. Such a scenario can account for the non-detection of the compact core component. However, the observed properties of the extended radio source in FRB 20201124A are markedly different from those observed in the remnant phase of radio galaxies. First, the radio spectrum of the remnant of radio-loud AGN is much brighter at lower frequencies, with a very steep spectrum ( $\alpha \gtrsim 1$ above a few hundred megahertz), due to the exponential cutoff from rapidly cooling electrons. Furthermore, the physical size of the region and the total radio power associated with remnants are, respectively, about two and three orders of magnitude larger than observed in our case, allowing us to rule out this scenario.

\section{Appendix H: Chandra limits on X-ray counterparts of FRBs detected by FAST and SRT}

We organized simultaneous coverage of the Chandra observation with FAST and SRT observations with the aim of searching for X-ray counterparts of FRBs. The Chandra observation took place on April 20 from 5:28 to 13:50 UT, lasting for $8.3 \mathrm{hrs}$.

The SRT (Bolli et al. 2015; Prandoni et al. 2017) observed FRB 20201124A on April 20, 2021, for 3 hours starting at 10:30 UT. Observations were performed at both $1.5 \mathrm{GHz}$ and $330 \mathrm{MHz}$. Data at $1.5 \mathrm{GHz}$ were recorded with the Australia Telescope National Facility (ATNF) Pulsar Digital Filter Bank (PDFB3) digital backend ${ }^{3}$, in search mode over an effective bandwidth of $420 \mathrm{MHz}$ split into $1 \mathrm{MHz}$ channels. Total intensity data were two-bit sampled every $100 \mu$ s. Data at $330 \mathrm{MHz}$ were recorded with the Reconfigurable Open Architecture Computing Hardware (ROACH1) digital backend in baseband mode over a bandwidth of $64 \mathrm{MHz}$, which was subsequently split into $0.25 \mathrm{MHz}$ channels. The raw data were coherently de-dispersed (removing the intra-channel smearing only) at the DM of the FRB (DM $=413 \mathrm{pc} \mathrm{cm}^{-3}$, as quoted by, e.g., CHIME/FRB Collaboration 2021b), and the resulting total intensity data were eight-bit sampled every $128 \mu \mathrm{s}$. A search for single pulses was performed on the data using the SPANDAK

\footnotetext{
3 http://www.srt.inaf.it/media/uploads/astronomers/ $\mathrm{dfb} \cdot \mathrm{pdf}$
} 
Table H.1. Limits on X-ray burst energy associated with FRBs.

\begin{tabular}{lcccc}
\hline \hline FRB & $\mathrm{E}_{X R B}$ & $\mathrm{E}_{X R B, N}$ & $\mathrm{~N}_{F R B}$ & Ref. \\
\hline & {$[\mathrm{erg}]$} & {$[\mathrm{erg}]$} & & \\
\hline $20121102 \mathrm{~A}$ & $\lesssim 4 \times 10^{46}$ & $\lesssim 4 \times 10^{45}$ & 10 & 1 \\
$20180916 \mathrm{~B}$ & $\lesssim 1.6 \times 10^{45}$ & $\lesssim 1.6 \times 10^{45}$ & 1 & 2 \\
$20201124 \mathrm{~A}$ & $\lesssim 6 \times 10^{45}$ & $\lesssim 1.1 \times 10^{44}$ & 49 & 3 \\
\hline
\end{tabular}

References. (1) Scholz et al. (2017); (2) Scholz et al. (2020); (3) this work

pipeline $^{4}$ (Gajjar et al. 2018). The pipeline uses RFIFIND from the PRESTO package ${ }^{5}$ (Ransom 2001) for high-level RFI excision. It then performs a first search for bursts through HEIMDALL (Barsdell et al. 2012). A DM range from 0 to $1000 \mathrm{pc} \mathrm{cm}^{-3}$ was searched for at $1.5 \mathrm{GHz}$ using a maximum window size for matched filtering of $130 \mathrm{~ms}$. In the case of the coherently dedispersed $330 \mathrm{MHz}$ data, a finer search was performed in the DM range $400-450 \mathrm{pc} \mathrm{cm}^{-3}$, with a maximum window size of 260 $\mathrm{ms}$. The pipeline finally performs sifting of the de-dispersed data and produces plots for the surviving candidates. Each candidate found by HEIMDALL at DMs within the range $400-450 \mathrm{pc} \mathrm{cm}^{-3}$ was visually examined. In order to validate genuine candidates, we ran an ad hoc program with more sensitive RFI excision procedures optimized for SRT data. This program searches for the most corrupted frequency channels in the DM zero data using the spectral kurtosis algorithm, with $5 \sigma$ thresholding, as provided by the software package YOUR ${ }^{6}$ (Aggarwal et al. 2020). It then applies baseline subtraction and normalizes the data for the average bandpass. A check for possible corrupted temporal bins due to the presence of impulsive RFI is then performed with interquantile range mitigation, similar to in Rajwade et al. (2020). Finally, it de-disperses the data to the derived DM and smooths them via a 2D Gaussian filter. A single candidate not resembling RFI was found in the data at $1.5 \mathrm{GHz}$ at barycentric time 10:44:13.815 TDB (reported at infinite frequency) at a DM compatible with that of FRB 20201124A: DM $=421 \pm 4 \mathrm{pc} \mathrm{cm}^{-3}$. The burst had a signal-to-noise ratio of $S / N=27.3$ and a width of $W=10 \mathrm{~ms}$, resulting in a fluence of $F=13 \pm 3 \mathrm{Jy} \mathrm{ms}$. Since the data are uncalibrated, the estimated fluence of the burst was calculated using the modified radiometer equation (see, e.g., Lorimer \& Kramer 2004), adopting an antenna gain of $0.55 \mathrm{~K} / \mathrm{Jy}$ and a system temperature of $30 \mathrm{~K}$. No burst was observed in the data at $330 \mathrm{MHz}$ either simultaneously with the burst at $1.5 \mathrm{GHz}$ (with the time series both shifted to infinite frequency in order to remove the delay introduced by the DM at the two different frequencies) or at any other time during the observing session, down to a limiting fluence of $7 \mathrm{Jy} \mathrm{ms}$.
FAST carried out a 55-day observational campaign starting from April 1, 2021, soon after the CHIME alert (CHIME/FRB Collaboration 2021b). More than 1600 FRB bursts were detected in the $1.0-1.5 \mathrm{GHz}$ frequency band. The details of FAST observations will be reported in a separate paper (H. Xu et al. 2021, in preparation). During the Chandra observation, FAST observed for 2 hours (8:00-10:00 UT), detecting 48 bursts with fluences in the range 0.017-5.5 Jy ms (median $=0.22$ $\mathrm{Jy} \mathrm{ms}$; average $=0.65 \mathrm{Jy} \mathrm{ms}$ ) and with durations from $4 \mathrm{~ms}$ to 49 $\mathrm{ms}$ (median=14 ms; average $=19 \mathrm{~ms}$ ).

We searched for coincidences with the three X-ray photons detected by Chandra. The X-ray photon arrival times were barycenter corrected using the barycorr task within HEASoft v. 6.27.2. The closest FRB-X-ray photon time difference is $314 \mathrm{~s}$. Considering the number of bursts and the count rate, the probability of a random association is close to $100 \%$, and thus we can exclude a significant coincidence with an FRB. We derived a $5 \sigma$ upper limit on the X-ray fluence of the FRB as follows (Scholz et al. 2017). For an X-ray burst arriving at the time of an FRB, we obtain an upper limit of 14 counts (at 5 sigma; Kraft et al. 1991), which corresponds to an upper limit of $\mathrm{F}(2-10 \mathrm{keV})=2.5 \times 10^{-10} \mathrm{erg} \mathrm{cm}^{-2}$ on the fluence, and of $\mathrm{E}=6 \times 10^{45} \mathrm{erg}$ on the X-ray burst energy, assuming the X-ray spectrum described in Appendix B. As the Chandra background is negligible, this limit is independent of the duration of the FRB up to the difference in arrival time of the closest Chandra photon (Scholz et al. 2017). In order to derive the upper limit for an X-ray burst occurring at a time later than $314 \mathrm{~s}$ after an FRB, we considered the number of trials and derived the singletrial confidence level, which was used to compute the upper limit following the prescription of Kraft et al. (1991). The number of trials is equal to the duration of the observation divided by the flare duration. Assuming a typical duration of $19 \mathrm{msec}$, comparable to the radio burst, and considering the negligible background, we derive an upper limit of 29 counts, corresponding to a fluence of $\lesssim 5 \times 10^{-10} \mathrm{erg} \mathrm{cm}^{-2}$ and an energy of $\mathrm{E} \lesssim$ $1.6 \times 10^{46} \mathrm{erg}$. Under the assumption that X-ray bursts are emitted for each of the 49 radio bursts, we can stack individual limits to derive an upper limit of $0.3 \mathrm{cts}$. The corresponding upper limits on the fluence and energy of the putative X-ray bursts are thus $5 \times 10^{-12} \mathrm{erg} \mathrm{cm}^{-2}$ and $E_{\mathrm{X}} \lesssim 1.1 \times 10^{44} \mathrm{erg}$. This corresponds to an upper limit on the ratio of the flare energy in $\mathrm{X}$-rays to radio of $E_{\mathrm{X}} / E_{\mathrm{R}} \lesssim 5 \times 10^{5}$, and to a radio-to-X-ray spectral index of $\alpha_{R X} \gtrsim 0.4\left(\mathrm{~F}(v) \propto v^{-\alpha}\right)$. In Table H.1 we compare the limits derived on the X-ray burst associated with FRBs with two repeating FRBs: FRB 20121102A(Scholz et al. 2017) and FRB 20180916B (Scholz et al. 2020). Overall, the most constraining observations for X-ray bursts in coincidence with FRB is $E_{\mathrm{X}} \lesssim 1.6 \times 10^{45} \mathrm{erg}$ for a single burst in FRB $20180916 \mathrm{~B}$ and $E_{\mathrm{X}} \lesssim 1.1 \times 10^{44} \mathrm{erg}$ in FRB $20201124 \mathrm{~A}$, assuming that all FRBs have an associated $\mathrm{X}$-ray burst.

\footnotetext{
4 https://github.com/gajjarv/PulsarSearch

https://github.com/scottransom/presto

6 https://github.com/thepetabyteproject/your/
} 